almost 2 times more likely to experience occupational injury than the White comparison group (pooled RR: 1.79, 95\% CI 1.65 to 1.93$)$.

Conclusions Differences in race increase the risk of experiencing workplace injuries. The causes of these discrepancies could vary greatly by occupation type. Future studies should summarize injury rates by occupation to allow for understanding of risks minority employees face.

Significance and Contributions to Injury and Violence Prevention Science It is important to understand the risks associated with injuries within the workplace that are making occupational environments less safe for minority workers. Understanding these risks allow for the development of innovative preventative methods to ensure all workers safe working environments.

\section{EXAMINATION OF THE IMPACT OF RECURRENT OVERDOSE AND MEDICATION-ASSISTED TREATMENT ON AFFECTING MORTALITY AMONG WEST VIRGINIA MEDICAID BENEFICIARIES}

Zheng Dai. West Virginia University

10.1136/injuryprev-2020-savir.121

Statement of Purpose The study aims to explore the trend of survival rates associated with recurrent overdose, and evaluate the effectiveness of MAT among the West Virginia (WV) Medicaid population.

Methods The primary outcome events include recurrent overdose and death. The occurrence of first nonfatal overdose was identified and an overdose cohort was then determined to allow 12 months of observation after the event. Kaplan-Meier method and log-rank test were used to assess the difference of time-to-events between subgroups.

Results A total of 446565 (87\%) continuously enrolled WV Medicaid beneficiaries from 2014 to 2016 were analyzed in the study. The overdose cohort included 2081 subjects, and $261(12.5 \%)$ subjects experienced recurrent overdose. Only 483 subjects received MAT other than methadone, and approximately 29\% ( $=139)$ of them initiated MAT after the index overdose. People who experienced a recurrent overdose in the 12 month follow the index overdose had a rapidly decreased survival rates, compared with those who did not $(p=0.0123)$. For subjects who received any MAT and those who had longer duration receiving MAT were both significantly associated with a higher survival rates $(\mathrm{p}=0.0398$ and 0.0458). Surprisingly, people who received any MAT were associate with a non-significantly higher incidence rates of recurrent overdose. This situation was further compounded by the observation that an early initiation of MAT after the index overdose was associated with a non-significantly lower survival rates.

Conclusion Recurrent nonfatal overdose increases mortality risk. Although MAT has been proven to be effective in preventing death, many subjects received MAT too late to be saved from another overdose event.

Significance Findings from this study will provide compelling evidence to inform MAT expansion and policy reform efforts in West Virginia to timely deliver MAT to fulfill patients' urgent needs.

\section{THE RELATIONSHIP OF SELF-REPORTED RESILIENCE AND TRAUMATIC CALL EXPOSURE AMONG PATROL OFFICERS AT THE DALLAS POLICE DEPARTMENT}

Alaina Beauchamp. University of Texas Health Science Center at Houston School of Public Health

\subsection{6/injuryprev-2020-savir.122}

To characterize law enforcement officer (LEO) resilience to physiological and psychological stress and describe the impact of cumulative traumatic exposure on resilience.

Patrol officers at five of the Dallas Police Department divisions volunteered to participate in 1.5 hour semi-structured focus groups. Focus groups $(n=18$ LEO) assessed experiences with traumatic calls and cumulative stress over the course of a shift. Transcripts were thematically analyzed in an iterative deductive/inductive coding scheme to identify anticipated and emergent findings.

The majority of participants were male and ages ranged from 23 to 61 years (mean=38 years). Participants were 67\% non-Hispanic white and $56 \%$ had a four-year college degree. Officer resilience emerged in many forms, including officernominated local innovations and immediate and distal coping mechanisms. Resilience to traumatic calls over time was significantly influenced by tenure. For experienced LEOs ( $>10$ years of service), a reduction in adrenaline reaction was recognized for high-stress calls similar to past encounters. Participants describe a peak of perceived adrenaline rush, which they subsequently become resilient to reaching during similar circumstances.

The frequency at which LEO respond to high-stress calls was described as a method of instinctively building resilience to situational stress reactions. This suggests that previous LEO experiences could have an integral part in influencing the physiology and psychology of LEO stress reactions on the job. The themes gathered serve to inform future areas of the study, as well as a predictive algorithm that can build in time between high stress calls to improve LEO occupational health and safety.

LEO experience stressful calls for service on a daily basis, which may cumulatively compound placing the LEO and civilians at risk of unintended consequences, like PTSD, injury, or mortality. To our knowledge, the degree of occupational stress resilience among LEO has not been considered for intervention in the cumulative stress pathway.

\section{SOCIODEMOGRAPHIC CHARACTERISTICS AND ENVIRONMENTAL FEATURES OF MASS-SHOOTING LOCATIONS IN TEN US CITIES}

Sharmaine Ross, Steven Forman, Christopher Morrison, Sara F Jacoby, Beidi Dong, Zoë Maher, Jessica H Beard. Lewis Katz School of Medicine at Temple University; Department of Epidemiology, Columbia University; School of Nursing, University of Pennsylvania; George Mason University

\subsection{6/injuryprev-2020-savir.123}

Statement of Purpose Mass-shootings involving $\geq 4$ people injured or killed occur frequently in cities in the United States. However, little is known about the places affected by mass-shootings. We aimed to describe the sociodemographic 
characteristics and environmental features of mass-shooting locations in US cities.

Methods Using Gun Violence Archive data, we identified all mass-shootings in the 10 US cities with the highest homicide rates from 2014-2017 $(n=214)$. We geocoded the event locations and used American Community Survey estimates to describe the sociodemographic characteristics of mass-shooting block groups with 6 indicators of structural disadvantage. We compared mass-shooting block group sociodemographic characteristics with each city's characteristics overall using Students t-tests. We assessed the environmental features of mass-shooting locations using Google Street View to code 60 elements of the visible environment.

Results Compared to overall city demographics, mass-shooting block groups had significantly higher rates of poverty $(28.0 \%$ vs. $35.1 \% ; \mathrm{p}=0.004)$, unemployment $(8.2 \%$ vs. $10.1 \%$; $\mathrm{p}=0.005)$, Black residents $(47.5 \%$ vs. $70.0 \% ; \mathrm{p}=0.012)$ and renter occupied units $(53.0 \%$ vs. $57.9 \%$; $\mathrm{p}=0.027)$, while percentage of college attendees/graduates $(55.8 \%$ vs. $30.2 \%$; $\mathrm{p}<0.0001)$, and median household income $(\$ 37,302$ vs. $\$ 31,313 ; p=0.009$ ) were significantly lower in mass-shooting block groups. Sixty-four percent of mass-shootings occurred in residential locations, and most locations demonstrated indicators of physical disorder. Average building conditions were graded as moderate; $37.4 \%$ of locations had buildings with broken/boarded windows and $78.3 \%$ had moderate to extreme littering. $94.3 \%$ and $93.1 \%$ of locations had no parks or playgrounds respectively.

Conclusions Mass-shootings in urban environments tend to occur in residential locations with significant structural disadvantage. The built environment of mass-shooting locations is characterized by blighted buildings and limited green spaces.

Contributions to Injury and Violence Prevention Science Discourse about mass shootings often excludes urban spaces. Structural disadvantage and modifiable environmental features are key targets for mass-shooting prevention in US cities.

\section{ASSOCIATED RISKS FOR FALLS AMONG ADULTS AGES 65 AND OLDER: INSIGHTS FROM THE NATIONAL HEALTH INTERVIEW SURVEY, 2015-2017}

Matthew Garnett, Holly Hedegaard, Merianne Spencer. Centers for Disease Control and Prevention

\subsection{6/injuryprev-2020-savir.124}

Statement of Purpose This analysis uses data from the National Health Interview Survey (NHIS), a national in-person survey of non-institutionalized adults, to examine risk factors among adults aged 65 and older who reported a fall.

Methods/Approach Data from the NHIS was used to identify older adults who experienced a fall-related injury in the 90 days prior to survey participation. Analysis was conducted using data from all older adults who reported a fall and on the subset of adults who had a fall that required emergency department (ED) treatment or hospitalization. The study used aggregate data from 2015-2017. Established methodologies to account for the complex sampling and survey design were employed to generate national estimates. Bivariate (X2 tests) and multivariate (logistic regression) analyses were conducted to examine differences by demographic characteristics and comorbid medical conditions.
Results During the 3-year period, there were 939 older adult survey participants who reported a fall in the past 90 days (national estimate $=1,020,779$ ). Of these, 534 were hospitalized or treated at an emergency department (ED) (national estimate $=589,251$ ). Falls were significantly associated $(p<0.05)$ with increasing age, being female, being white-non-Hispanic, and being widowed. After controlling for demographic factors, comorbid conditions that limited daily activities/functioning and were significantly associated with falls included: depression/anxiety, dementia, cancer, arthritis, diabetes, lung problems (asthma and other breathing conditions), and being obese. Similar findings were observed for severe falls that required hospitalization or ED treatment.

Conclusions The NHIS provides insight into the risk of falls among older adult populations. Disparities exist across demographic groups, and key co-morbidities may affect fall risk among older adults.

Significance/Contributions Studies examining falls among older adult populations are often limited to examining health outcomes or disease burden. This analysis adds contextual information on possible risk factors for falls using nationally representative survey data.

\section{FUN RIDE OR RISKY TRANSPORT: GOLF CART-RELATED INJURIES TREATED IN U.S. EMERGENCY DEPARTMENTS FROM 2007 THROUGH 2017}

Rebecca McAdams, Kyle Horvath, Kristin Roberts, Motao Zhu, Lara McKenzie. Center for Injury Research Policy at the Abigail Wexner Research Institute at Nationwide Children's Hospital

\subsection{6/injuryprev-2020-savir.125}

Purpose Golf cart-related injuries are a source of morbitiy for people of all ages, especially children, and their use has increased in recent years. However, design modifications or legislation to reduce the overall burden of these injuries have been nonexistent. This study sought to characterize the epidemiology of golf cart-related injuries treated in United States (U.S.) hospital emergency departments (EDs).

Methods/Approach We performed a retrospective analysis by using the National Electronic Injury Surveillance System data for patients of all ages treated in EDs (2007-2017) for a golf cart-related injury. Bivariate comparisons were conducted by using design-adjusted chi-square tests, and the strength of association was assessed by relative risks (RR) with corresponding 95\% confidence intervals (CIs). Injury rates over time were analyzed by using linear regression. We generated national estimates from 3567 cases.

Results From 2007-2017, an estimated $156 \quad 040 \quad$ (95\% $\mathrm{CI}=102$ 402-209 679) patients were treated in U.S. EDs for golf cart-related injuries. The average rate of traumatic brain injuries (TBIs) in children (1.62 per 100000 children) was more than three times that of TBIs in adults $(0.52$ per 100 000 adults; rate ratio $=3.12 ; 95 \% \mathrm{CI}=3.05-3.20) 1.46$ times that of TBIs in seniors (1.11 per $100 \quad 000$ seniors; 95\% $\mathrm{CI}=1.42-1.51)$. The injury rate in seniors increased significantly by $67.6 \%$ from 4.81 per 100000 seniors in 2007 to 8.06 per 100000 seniors in 2017 (slope $=0.096$; $\mathrm{p}=0.041$ ). Conclusions Golf cart use remains an important source of injury for people of all ages, especially children. As use continues to increase, it is unlikely that golf cart-related injuries 